\title{
EMPLEO DE SEMILLAS COMO INGREDIENTE SACIANTE: CHÍA Y SÉSAMO
}

The use of seeds as satiating ingredient: chia and sesame

\section{Gil-Gallego, M.T.; Planes-Muñoz, D.; López-Nicolás, R* .}

Departamento de Tecnología de los Alimentos, Nutrición y Bromatología. Facultad de Veterinaria. Campus Internacional de Excelencia “Campus Mare Nostrum”. 30100, Espinardo (Murcia). España

* Autor para correspondencia: López-Nicolás, Rubén. Departamento de Tecnología de los Alimentos, Nutrición y Bromatología. Facultad de Veterinaria. Campus Internacional de Excelencia “Campus Mare Nostrum”. 30100, Espinardo (Murcia). España. E-mail: rubenln@um.es

Historial del artículo:

Recibido: 28 de enero, 2020

Aceptado: 6 de octubre, 2020

\section{RESUMEN}

Debido al gran aumento de la prevalencia del sobrepeso y obesidad en todo el mundo, ha aumentado el interés de estudio de los mecanismos que llevan a la población a un desequilibrio energético, aumentando su peso corporal de forma excesiva. El control de la ingesta de energía va determinado por la percepción de la saciedad durante y después de las comidas. Hay ciertos alimentos que por sus características nutricionales (cantidad de fibra, hidratos de carbono, proteína y grasa) pueden influenciar de forma positiva sobre el apetito (disminuyendo el deseo por la comida). Por ello sería una buena estrategia nutricional ver cuáles de los alimentos presenta mayor relación sobre la saciedad. Realizamos un ensayo clínico cruzado aleatorizado con un periodo de lavado de 5 días, como mínimo. El objetivo del presente estudio fue evaluar el efecto de las semillas de sésamo y chía (por su gran cantidad de fibra soluble) sobre la saciedad (introducidas junto con yogur en el desayuno). La muestra estaba formada por 18 voluntarios. Cada voluntario realizó los 3 tipos de desayunos de forma aleatoria: (café con leche o té con leche + tostada con aceite de oliva y sal) + (yogur, yogur con chía o yogur con sésamo). Antes del desayuno tuvieron que rellenar una encuesta sobre apetito (VAS) y después 7 más (a los 15, 45, 75, 105, 135, 165 y 195 minutos tras el desayuno). Para valorar el efecto saciante de las semillas, se analizaron las preguntas del VAS que presentan relación directa con la saciedad, y se realizó un ANOVA. Los resultados mostraron que no había diferencias estadísticamente significativas ( $p>0.05)$ entre las semillas estudiadas.

Palabras clave: saciedad, obesidad, fibra, dieta Mediterránea 


\begin{abstract}
Nowadays, there is a high prevalence of overweight and obesity around the world, so the interest in studying the mechanisms that lead the population to an energy imbalance has increased. The control of energy intake is determined by the perception of satiety during and after meals. There are certain foods that, due to their nutritional characteristics (amount of fibre, carbohydrates, protein and fat), can positively influence the appetite (decreasing the desire to eat). Thus, it would be a good nutritional strategy to see which foods have a greater satiety effect. We performed a randomized crossover clinical trial with at least 5-days washout period. The objective of the present study was to evaluate the effect of sesame and chia seeds (due to their large amount of soluble fibre) on satiety. The sample consisted of 18 volunteers. Each one performed the 3 types of breakfasts randomized: (coffee with milk or tea with milk + toast with olive oil and salt) + (yoghurt, yoghurt with chia or yoghurt with sesame). Before breakfast they had to fill out an appetite survey (VAS) and then 7 more (at 15, 45, 75, 105, 135, 165 and 195 min after breakfast). To assess the satiating effect of the seeds, the questions of the VAS directly related to satiety were analysed, and ANOVA was performed. The results showed that there were no statistically significant differences $(\mathrm{p}>0.05)$ between seeds.
\end{abstract}

Keywords: satiety, obesity, fibre, Mediterranean diet.

\section{INTRODUCCIÓN}

En estos últimos años, se ha visto un desequilibrio entre el consumo y gasto energético, y como consecuencia han aumentado los niveles de sobrepeso y obesidad, así como las enfermedades relacionadas con dichos estados (Buckland et al., 2008). Por todo ello han aumentado las investigaciones sobre las estrategias nutricionales para prevenir dicho consumo excesivo de energía. Puesto que el uso de tratamiento farmacológicos y complementos alimenticios con componentes bioactivos no son eficaces o presentan muchos efectos adversos, se está estudiando los componentes dietéticos naturales que interaccionan sobre el apetito $\mathrm{y}$ la saciedad, con el fin de disminuir la ingesta calórica de la dieta, los niveles de obesidad y sus enfermedades relacionadas (Buckland et al., 2008).

Según la OMS, "La obesidad y sobrepeso se define como una acumulación anormal o excesiva de grasa que puede ser perjudicial para la salud" (Organizacion Mundial de la Salud (OMS), 2012), debiéndose a un balance ener- gético positivo (se consume más de lo que se gasta)(Amin \& Mercer, 2016). Estos conceptos también los podemos definir usando el índice de masa corporal (IMC) expresado en $\mathrm{kg} / \mathrm{m}^{2}$, estando por encima de 30 en la obesidad y por encima de 25 en el sobrepeso (Organizacion Mundial de la Salud (OMS), 2012).

Desde el 1975 hasta ahora ha ido aumentando la prevalencia de dichos estados de acumulación de grasa, hasta tal punto que la OMS la considera como la Epidemia del siglo XXI, afectando gravemente en la salud de las personas (Verónica \& Vliegenthart, 2003).

Algunas de las causas de esta epidemia es el cambio de factores genéticos, factores hormonales, factores socioeconómicos y lo más importantes, el cambio del estilo de vida y los hábitos alimentarios (Buckland et al., 2008). En el último siglo se han producido diversos cambios en la agricultura, producción, almacenamiento y en el procesado de los alimentos, dando lugar a la gran variedad de alimentos baratos, sabrosos, de elevada densidad energética y con un elevado contenido en grasa y azúcar que hay en la actualidad. La población ha pasado de una 
dieta mediterránea (rica en frutas, verduras y legumbres) a una dieta más procesada y con mayor densidad energética (Buckland et al., 2008; Amin \& Mercer, 2016). Este cambio tan rápido en la industria ha fomentado un ambiente obesogénico, haciendo imposible la adaptación de las personas. Finalmente, esto ha llevado a que el sistema de control del apetito no pueda evitar consumir un exceso de energía (Buckland et al., 2008; Halford \& Harrold, 2012).

Por todas estas razones se han realizado estudios centrados en la medición del hambre, del apetito, del deseo de comer e incluso de la sensación de llenado o plenitud tras la ingesta, estableciendo correlaciones entre los factores biológicos y psicológicos que nos ayudan a comprender el comportamiento alimentario de cada individuo. Este comportamiento influye mucho en la sensación de saciedad de cada persona, por ello, cada vez se están realizando más estudios acerca de la saciedad (García-Flores et al., 2017).

El fenómeno de la saciedad es un concepto complejo que incluye desde factores preingesta, como los psicológicos, sensoriales e incluso sensaciones aprendidas con las experiencia; hasta post-ingesta (volumen, densidad energética y contenido en macronutrientes, entre otras); e incluso post-absortivas (aminoácidos, glucemia, hormonas saciantes, etc). Todo ello hace difícil un abordaje completo y hay que enfocarlo pormenorizadamente en cada parcela.

En el caso directo de medir la inhibición de la ingesta posterior tras una comida, se puede cuantificar midiendo la duración del intervalo que separa el fin de una comida con el comienzo de la siguiente, de esta manera podemos medir la saciedad como un período de tiempo. También podemos medir como disminuye la ingesta en el próximo episodio alimentario tras un intervalo fijo entre comidas, aquí se presenta la inhibición de la ingesta como una reducción de las cantidades. Otra manera, es medir las variaciones en la intensidad de varias sensaciones subjetivas tras la ingesta de una comida, en esta ocasión, la saciedad se presenta como una puntuación (Tremblay \& Bellisle, 2015). Entre ésta ultima opción, encontramos las escalas más usadas y con mayor relevancia en estudios de saciedad, que son las escalas analógicas visuales (VAS, procedente del inglés Visual Analogue Scale). Éstas evalúan la intensidad de la sensación de la saciedad y generan perfiles de saciedad muy repetidos tras las mismas ingestas de una comida. Las puntuaciones de la escala VAS se registran antes e inmediatamente después de una comida, además de posteriores intervalos regulares (cada 15-30 minutos hasta 1 hora) durante 3-5 horas o hasta el comienzo de la próxima ingesta. Las escalas VAS son fácilmente interpretadas por los sujetos incluidos en los estudios, poseen gran fiabilidad, sensibilidad y son idóneas para el manejo matemático y estadístico (Blundell et al., 2010; Tremblay \& Bellisle, 2015).

Como se ha mencionado anteriormente, son muchos los factores nutricionales (composición de macronutrientes de los alimentos, densidad energética, cantidad y tipo de fibra e índice y carga glucémica) y no nutricionales (tamaño de porción, palatabilidad) relacionados con la saciedad. En cuanto a la composición de macronutrientes, las dietas elevadas en proteínas (30\% de proteínas, $30 \%$ de grasa y $40 \%$ de carbohidratos) han demostrado su eficacia en la pérdida de peso y para no recuperarlo después. Sin embargo, se cuestiona su seguridad y no hay un consenso sobre qué tipo y cantidad de proteína es mejor para el control del apetito a medio y largo plazo (Montmayeur \& Coutre, 2009; Halford \& Harrold, 2012).

Por otro lado, hay muchas evidencias de la relación entre la fibra dietética y la prevención de las enfermedades cardiovasculares (ECV), relacionado a su vez con el índice glucémico (IG) y la carga glucémica (GL) en pacientes con hiperlipidemia o diabetes. Puede ser útil la utilización de la fibra para el tratamiento de la obesidad, pues agrega volumen al tamaño de la porción del producto, reduce la densidad de 
energía de una comida, y todo ello mejora el control del apetito mediante el retraso del vaciado gástrico, así como aumentando las señales de saciedad post-ingesta. Además, también hay que tener en cuenta el tipo de fibra para el estudio de la saciedad, ya que la fibra soluble (pectinas, gomas, mucílagos, inulina, rafinosa, fructo-oligosacáridos y almidón resistente) proporciona viscosidad a los alimentos, absorbe agua con facilidad y forma geles viscosos que retardan el vaciado gástrico, siendo además fáciles de fermentar a lo largo del intestino y por lo tanto, proporcionando un efecto mayor de plenitud que la fibra insoluble (Montmayeur $\&$ Coutre, 2009).

En relación a las grasas y el apetito es importante mencionar la importancia del grado de insaturación lipídica. Se ha visto que ácidos grasos con la misma longitud de cadena se establece una relación donde a mayor grado de insaturación (mayor número de dobles enlaces) mayor saciedad (Dernini \& Berry, 2015).

La combinación de cantidades pequeñas de proteína y grasas insaturadas, junto con fibra, presenta un potencial para inducir la saciedad (reduciendo el apetito, el deseo de comer y el consumo posterior, a la vez que se aumenta la sensación de plenitud) (Halford \& Harrold, 2012). Todas estas condiciones nutricionales, quedan reflejadas en la dieta mediterránea (DM), que ha sido descrita como un modelo de alimentación saludable asociado con importantes beneficios nutricionales y para la salud, como, por ejemplo; su papel protector para las enfermedades cardiovasculares (Bach-Faig et al., 2011; Carbonneau et al., 2017). Desde los años 60 hasta hoy, la dieta mediterránea se ha erosionado progresivamente debido a la amplia difusión de la economía occidental, la cultura urbana impulsada por la tecnología, así como la globalización en la producción y consumo de alimentos procesados (Davis et al., 2015).

En este estudio nos vamos a centrar en el impacto saciante que presentan unos alimentos mediterráneos que contienen alta proporción de proteínas, fibra y grasas insaturadas, como son las semillas. Concretamente hemos estudiado las semillas de sésamo y su comparación con semillas de chía, cuyo efecto saciante ha sido ampliamente demostrado en la bibliografía (Ayaz et al., 2017; Vuksan et al., 2017). Las semillas de chía se componen de un $31 \%$ de grasas (siendo mayormente poliinsaturadas), $16-21 \%$ proteínas y un $40 \%$ de carbohidratos (siendo fibra el $30 \%$ y el otro $10 \%$ almidones complejos). La elección de las semillas de sésamo se hizo por ser una semilla típica de la región Mediterránea, siendo una fuente de lípidos (sobre todo en ácidos grasos poliinsaturados), fibra y proteína de alto valor biológico. Presentan un $55 \%$ grasa, $18-25 \%$ proteínas, $13.5 \%$ hidratos de carbono(3.2\% glucosa, $2.6 \%$ fructosa y el $7.7 \%$ restante es fibra), además de un perfil idóneo minerales y vitaminas. Además, contiene lignanos (sesamina y sesamolina), que junto con otros principios activos, presenta funciones fisiológicas beneficiosas para la salud (Pal, 2010; Asghar et al., 2014; Saeed et al., 2015). El posible inconveniente de este tipo de semillas es su alta alergenicidad, lo que hace imposible su recomendación a la población general, debiendo quedar excluidas aquellas personas que han reflejado alergia frente al sésamo.

En general, la mejora de la sensación de la saciedad puede hacer que aquellas personas que no puedan resistir a las señales de un entorno obesogénico presenten mayor bienestar y autocontrol sobre su conducta alimentaria, consumiendo así mayor cantidad de alimentos saludables y mejorar su estado de ánimo, además de conseguir una pérdida eficaz de peso (Halford \& Harrold, 2012).

La hipótesis principal de nuestro estudio es estudiar si la inclusión de este tipo de semillas en el desayuno (yogur + sésamo o yogur + chía) presenta un mayor efecto saciante, reduciendo en consecuencia la ingesta de alimentos a corto plazo. Con todo ello, el objetivo del presente estudio fue evaluar el efecto de las semillas de sésamo y chía sobre la saciedad. 


\section{MATERIAL Y MÉTODOS}

\subsection{Diseño del estudio}

Nuestro diseño de estudio fue, un ensayo clínico cruzado aleatorizado con un periodo de lavado de 5 días como mínimo (Blundell et al., 2010). Reclutamos 18 participantes y cada uno paso de forma aleatoria por los 3 tipos de desayuno, teniendo en cuenta el periodo de lavado de una semana aproximadamente. El lugar donde realizaban los desayunos era un lugar calmado y no se permitía el uso de móvil durante el desayuno. Los desayunos fueron ajustados a las necesidades calóricas de cada participante, para lo que calculamos las necesidades energéticas mediante la fórmula de Harris- Benedict. Según las recomendaciones del Ministerio de Sanidad Español sobre alimentación saludable, y avalado por multitud de estudios (Gibney et al., 2018), sugieren que un desayuno saludable debe incluir el $20 \%$ de la ingesta calórica diaria, por ello, el resultado obtenido de la fórmula de Harris-Benedict lo multiplicamos por 0.2 para calcular las calorías del desayuno.

El desayuno del estudio presenta una parte fija de igual cantidad para todos los participantes (tostada, café con leche o té con leche) y una parte variable (yogurt con o sin semillas), en función del gasto energético total de cada individuo:

- Parte fija (189.6 kcal): $150 \mathrm{ml}$ de leche semidesnatada con $50 \mathrm{ml}$ de café o $50 \mathrm{ml}$ de agua mineral con té +30 gramos de pan blanco con una cuchara pequeña de aceite de oliva virgen extra $(5 \mathrm{ml})$ y una pizca de sal.

- Parte variable: las cantidades de esta parte depende de las necesidades energéticas de cada participante, pero se utilizó como referencia 100 gramos de yogur +14 gramos de semillas de chía (Buckland et al., 2008). Esta cantidad de semillas se encuentra entre los 10-20 gramos recomendados al día según la Sociedad Española de Nutrición y Dietética. Puesto que los dos tipos de semi- llas no presentan la misma densidad energética (las de sésamo son más calóricas que la de chía), la cantidad de semilla se ajustaron para obtener desayunos isocalóricos (Buckland et al., 2008).

El yogur usado en el estudio fue blanco azucarado y comprado en un mercado local.

En la figura 1 se puede apreciar un esquema de los 3 tipos de desayuno.

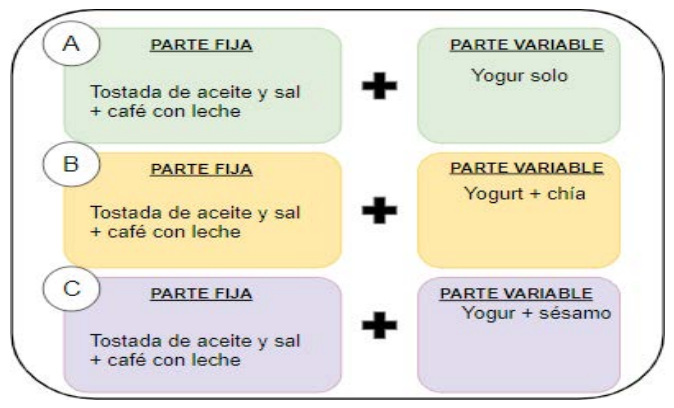

Figura 1: Tipos de desayunos.

\subsection{Voluntarios y comité de ética}

Se reclutaron 18 participantes para el estudio (8 hombres y 10 mujeres). Los criterios de inclusión fueron: edad entre los 23-53 años, índice de masa corporal (IMC) entre $21-30 \mathrm{~kg}$ / $\mathrm{m}^{2} \mathrm{y}$ con un peso corporal más o menos estable en los últimos tres meses, no ser diabéticos, ni hipertensos. Estos criterios son los descritos en la bibliografía para este tipo de estudios para poder obtener resultados fiables (Blundell et al., 2010). Los criterios de exclusión: ser intolerante a la lactosa, alérgico a algún alimento del estudio, ser celíaco, fumar (más de 5 cigarrillos/día), uso diario de medicamentos recetados (excepto medicamento para la hipertensión y anticonceptivos orales), embarazo o lactancia, índice de masa corporal (IMC) $>31 \mathrm{~kg} / \mathrm{m}^{2}$ o tener alguna enfermedad que afecte a la sensación de saciedad o apetito (como por ejemplo el síndrome de Prader Willi). Todos los participantes dieron su consentimiento por escrito después de 
haber recibido información verbal y por escrito sobre el estudio.

Nuestro estudio cuenta con la aprobación del comité de ética de la Universidad de Murcia (España) con el código 2051/2018. Antes del comienzo del estudio se les dio a los participantes la hoja de información y el consentimiento informado, el que entregaron firmado antes de la primera visita.

Los participantes fueron informados de que sus datos personales eran protegidos e incluidos en un fichero, con la garantía de la ley 15/1999, además de informarles de que podían abandonar el estudio en cualquier momento sin dar explicaciones y $\sin$ que ello le suponga perjuicio alguno.

\subsection{Instrumentos y variables}

Los instrumentos y las variables que hemos usado para la realización del estudio son:

- Tallímetro (marca SECA, número 213, procedencia España): altura (cm).

- Báscula (marca GRAM Group, modelo S36, procedencia España): para pesar los alimentos.

- Impedómetro (marca OMRON, modelo BF 511, procedencia Japón): peso (kg), IMC $\left(\mathrm{kg} / \mathrm{m}^{2}\right)$, porcentaje de grasa $(\%)$ y porcentaje de músculo (\%). A las personas con mayor IMC se le ha realizó el peso ajustado para el cálculo del gasto energético total.
Con la altura y el peso hemos calculado el GET, y con ello, la cantidad de yogur y semillas. En la tabla 1 se pueden ver las variables tomadas a cada uno de los voluntarios. - Encuestas: Escala Analógica Visual (VAS) del apetito y palatabilidad, PSS (Torres-Lagunas et al., 2015) (escala de estrés percibido, del inglés Perceived Stress Scale) y TFEQ (Three-Factor Eating Questionnaire). El Cuestionario de Alimentación de Stunkard y Messick (TFEQ) proporciona información acerca del comportamiento alimentario de las personas, tal como restricción y desinhibición ante la comida.

Con esta última encuesta podemos ver el comportamiento alimentario de cada participante, excluyéndolos en caso de presentar una puntuación muy elevada para alguna de los tres factores alimentarios evaluados. Además, los resultados de restricción, desinhibición y hambre nos sirven para ver si existe algún tipo de correlaciones con las demás variables tomadas (Clave, 1999; López-Aguilar et al., 2011).

En la Escala Analógica Visual (VAS) del apetito, las preguntas relacionadas con el hambre y la saciedad son (Blundell et al., 2010; Tremblay \& Bellisle, 2015):

- Hambre: ¿cómo de hambriento te sientes en este momento?

- Deseo de comer: ¿Cómo de fuerte es tu apetito por una comida? ¿Cómo de fuerte es tu apetito por un snack? ¿Cómo de fuerte es tu

Tabla 1. Descripción de la muestra

\begin{tabular}{|c|c|c|c|c|c|c|c|}
\hline Estadísticos & Edad & $\begin{array}{l}\text { Altura } \\
(\mathrm{cm})\end{array}$ & $\begin{array}{l}\text { Peso } \\
(\mathrm{kg}) \\
\end{array}$ & $\begin{array}{l}\text { IMC } \\
\left(\mathrm{kg} / \mathrm{m}^{2}\right) \\
\end{array}$ & $\begin{array}{l}\% \\
\text { Grasa }\end{array}$ & $\begin{array}{l}\% \\
\text { Músculo }\end{array}$ & $\begin{array}{l}\text { GET } \\
(\mathrm{Kcal}) \\
\end{array}$ \\
\hline Media & 31.8 & 167.5 & 75.7 & 26.3 & 34.7 & 32.5 & 2235.5 \\
\hline Mediana & 32.5 & 168 & 76 & 26 & 35 & 33 & 2228.6 \\
\hline Desviación estándar & 9.5 & 7.1 & 19.5 & 5.6 & 0.5 & 4.4 & 441.0 \\
\hline Máximo & 53 & 177 & 95 & 33.6 & 41 & 37 & 3028.5 \\
\hline Mínimo & 18 & 159 & 56 & 20 & 28 & 27 & 1584.8 \\
\hline
\end{tabular}

Estadísticos descriptivos de la muestra seleccionada para el estudio 
apetito por algo salado? ¿Cómo de fuerte es tu apetito por algo dulce?

- Plenitud: ¿Cómo de lleno te sientes en este momento?

- Motivación para comer: ¿Cuanta comida sientes que podrías comer en este momento?

\subsection{Procedimiento}

El protocolo de actuación que hemos seguido en el estudio es el siguiente: al comienzo del estudio se repartieron las hojas de información y el consentimiento informado a los participantes. El día que realizaban los desayunos, los voluntarios tenían que venir en ayunas y sin haber realizado deporte el día anterior. El primer día del estudio, se pesa y mide a los participantes para calcular su gasto energético total (GET) y las cantidades de yogur y semillas correspondientes a cada uno. Antes de entregarles el desayuno debían de rellenar la primera encuesta sobre apetito (A1), tras la que disponen de 15 min para ingerir todo el desayuno proporcionado. Al finalizar el desayuno se les entregaba la segunda encuesta sobre apetito (A2) y la encuesta de palatabilidad (P1). A continuación se les suministra a los participantes las 6 encuestas restantes sobre apetito para rellenarlas a los 45, $75,105,135,165,195$ minutos tras la primera encuesta (Tabla 2). Los participantes no podrán comer nada hasta terminar la última encuesta y deberán contabilizar la cantidad de agua bebida. Además, el último día se les dio la encuesta PSS y TFEQ.

\subsection{Análisis de datos}

Los datos obtenidos para las preguntas sobre hambre, llenado, deseo de comer y consumo prospectivo para cada alimento y a cada tiempo de estudio se analizó mediante ANOVA de una vía, siendo el factor el tipo de desayuno (yogur, yogur más chía o yogur más sésamo). A través de los valores obtenidos de las encuestas VAS, se calcularon las áreas bajo la curva (AUC del inglés, area under the curve) usando el método trapezoidal, puesto que es un parámetro representativo de la saciedad, siendo el más usado en este tipo de estudios (Buckland et al., 2008; Sociedad Venezolana de Farmacología. et al., 2016). Los valores de AUC entre los diferentes desayunos y para cada pregunta de saciedad realizada, se compararon mediante ANOVA de una vía, siendo el factor el tipo de desayuno. Para establecer las posibles correlaciones se usó el coeficiente de correlación de Pearson o Spearman, para los datos que presentaban normalidad o no, respectivamente. Previo a los contrastes de medias, la normalidad y homocedasticidad de los datos fue corroborada mediante el test

Tabla2. Encuestas realizadas

\begin{tabular}{llll}
\hline Encuesta & Número de preguntas & Tipo de pregunta & Hora (min) \\
\hline A1 & 11 & Apetito & 0 \\
A2 y P1 & 11 y 6 & Apetito y palatabilidad & 15 \\
A3 & 11 & Apetito & 45 \\
A4 & 11 & Apetito & 75 \\
A5 & 11 & Apetito & 105 \\
A6 & 11 & Apetito & 135 \\
A7 & 11 & Apetito & 165 \\
A8 & 11 & Apetito & 195 \\
\hline
\end{tabular}

Características de las encuestas utilizadas en el estudio 
de Shapiro Wilk y el test de Bartlett. Para todos los análisis realizados se consideró significativo un p valor $<0.05$. El análisis estadístico de los datos se llevó a cabo utilizando un paquete de software estadístico R y RStudio para Windows (versión 3.6.0).

\section{RESULTADOS}

Los 18 voluntarios reclutados completaron los tres desayunos, aunque 5 de ellos tuvieron que ser eliminados para el análisis estadístico por presentar valores elevados en el cuestionario TFEQ, lo que implica un comportamiento alimentario desequilibrado. Los participantes presentaban $70 \pm 17 \mathrm{Kg}$ del peso con un IMC $25 \pm 5$, una altura de $168 \pm 9 \mathrm{~cm}$, un porcentaje de grasa corporal de $21 \pm 11$ y un porcentaje de músculo de $22 \pm 6.14$.
La figura 2 muestra las calificaciones VAS para el hambre, la sensación de plenitud, el deseo de comer y el consumo prospectivo de comida. Como se muestra en las gráficas, los datos del AUC de las puntuaciones VAS no indicaron ninguna diferencia significativa entre los 3 tipos de desayuno $(\mathrm{P}>0.05)$ en ninguna pregunta (hambre, plenitud, deseo de comer y consumo prospectivo).

La figura 3 muestra las calificaciones VAS para el hambre, el llenado, el deseo de comer y el consumo prospectivo. Se analizaron punto por punto y realizamos un ANOVA de los 3 últimos puntos de cada gráfica. Estos puntos eran los que más separación se apreciaba a simple vista, por lo que podría haber mayores diferencias significativas.

Finalmente, no hubo diferencias estadísticamente significativas $(\mathrm{P}>0.05)$ entre los 3 tipos

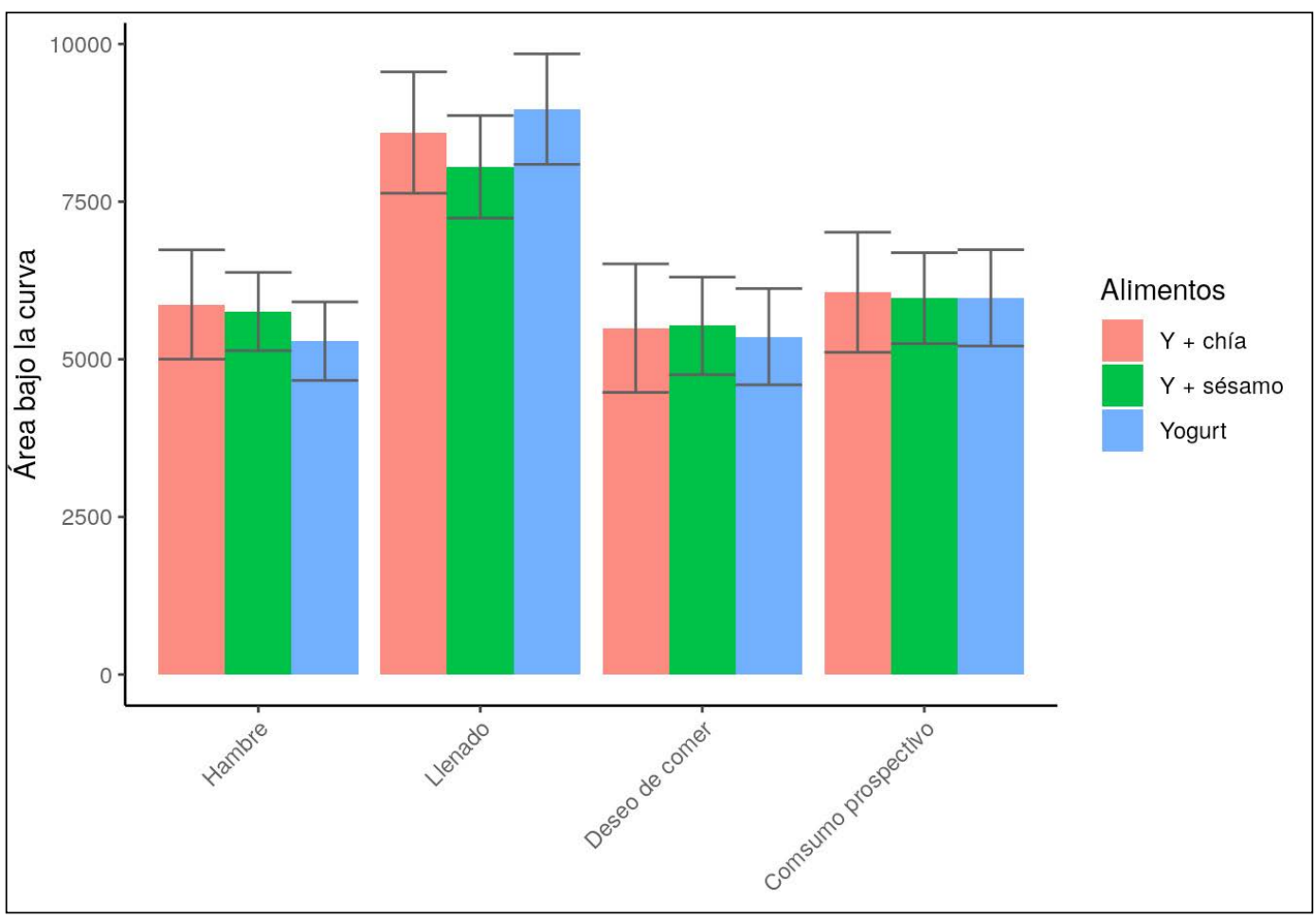

Figura 2. Resultados obtenidos tras analizar el AUC para cada pregunta del VAS. (Abreviaturas: Y: yogur) 


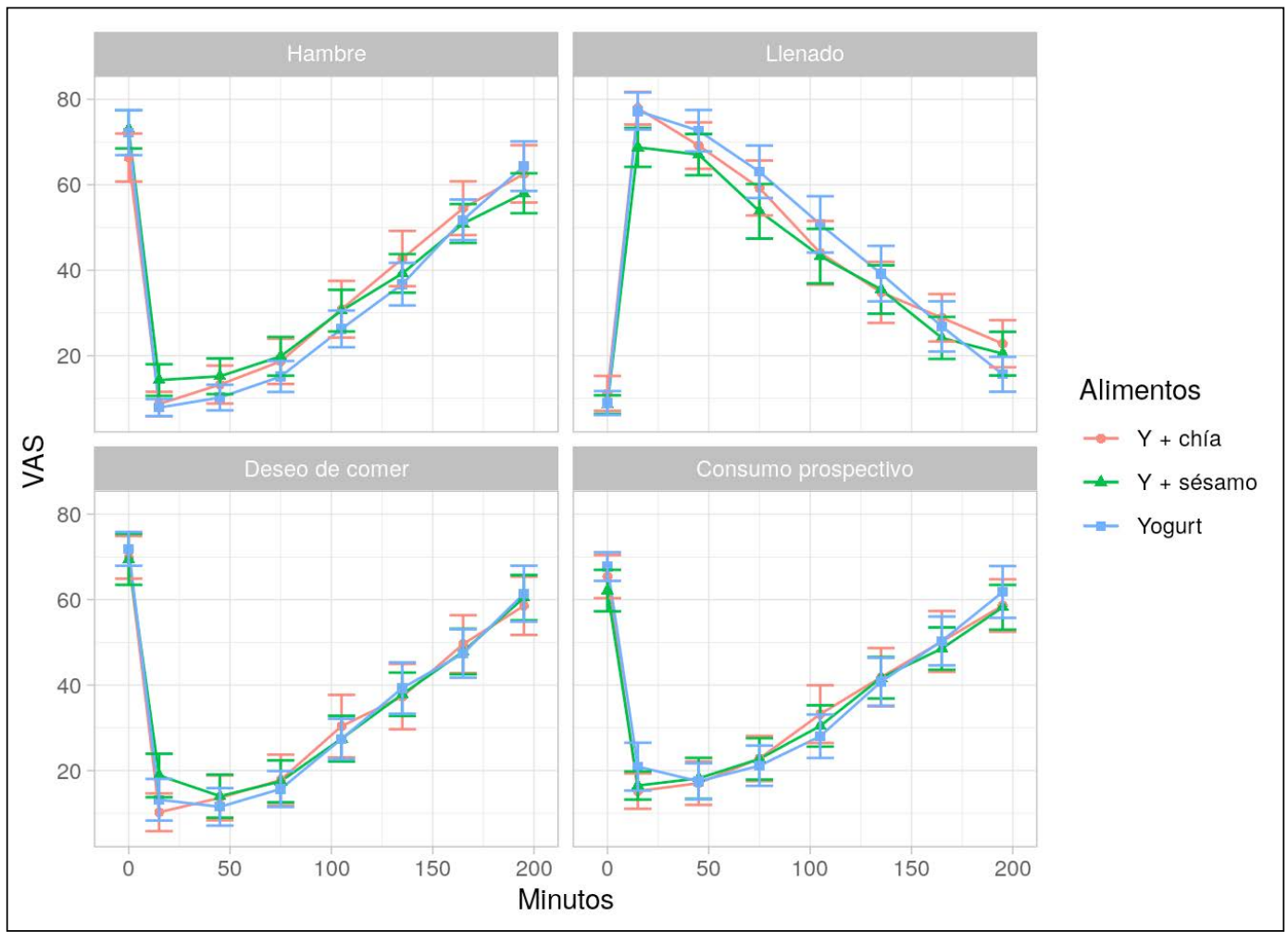

Figura 3. Resultados del análisis punto por punto de las preguntas del VAS. (Abreviaturas: Y: yogur)

de desayuno (chía, sésamo y yogur), siendo las puntuaciones del p-valor para cada pregunta los siguientes: 0.316 (hambre), 0.15 (sensación de llenado), 0.13 (deseo de comer) y 0.367 (consumo prospectivo).

De todas las correlaciones que realizamos entre las distintas variables, se representan en la tabla 3 las más significativas.

En cuanto a la escala de estrés percibido (PSS) pudimos ver que el nivel de estrés entre nuestros voluntarios no eran valores elevados, pudiendo agruparse en 3 bloques:

- El $11.1 \%$ de la muestra no presentó ningún tipo de estrés (sin estrés).

- El 55.6\% de la muestra presentó un nivel de estrés medio (estrés moderado).

- El 33.3\% de la muestra presentó estrés, pero no fueron niveles elevados.
En cuanto a los cuestionarios de comportamiento alimentario, los valores fueron normales para todos los voluntarios menos en 5 de ellos, que presentaron niveles elevado de restricción, desinhibición y hambre. Por ello estos no son candidatos fiables para establecer una relación objetiva sobre su sensación de saciedad, puesto que unos presentaban valores muy elevados de restricción (se restringen mucho a la hora de comer) y otros tenían valores muy elevados de desinhibición y hambre.

\section{DISCUSIÓN}

El presente estudio examinó los posibles efectos de la introducción de semillas de chía y sésamo en el desayuno sobre la saciedad. Los desayunos fueron ajustados (cantidad de yogur 
y semillas) dependiendo del gasto calórico de cada persona (20\% del gasto calórico total). Como resultado del estudio, no se encontraron diferencias significativas entre los 3 alimentos, lo que se pudo deber a diversos factores.

Por un lado, no se tuvo en cuenta el volumen gástrico, lo que propició una mayor ingesta de yogur en el desayuno que se tomaba solo, que cuando se incorporaban las semillas. En otros artículos similares (Vuksan et al., 2017) han demostrado el efecto del volumen de comida ingerida ya que las paredes del estómago se distienden estimulando los mecano-receptores que indican sensación de llenado, independientemente de la composición de macronutrientes ingeridos. Además, hay evidencias que relacionan la percepción de saciedad con la distensión gástrica, la cual puede estar inducida por hormonas intestinales como la colecistoquinina (CCK) o el péptido similar al glucagón 1 (GLP-1) (Carranza LE, 2016). Por este motivo, los resultados mostraron que, aunque ligeramente, cuando se ingiere el yogur sin semillas induce más saciedad y sensación de llenado; y menor sensación de hambre y consumo prospectivo de comida, que cuando se ingiere junto a semillas de sésamo o chía.
Por otro lado, no se ha tenido en cuenta el triturado de las semillas para romper la cáscara y permitir así una mayor gelificación. Se ha visto que las semillas de chía presentan una predisposición inherente para formar el proceso de gelificación al contactar con el agua, ya que están recubiertas por polisacáridos (glucosa, xilosa, ácido glucurónico, arabinosa, etc) solubles. Sin embargo, cuando se elimina la cubierta externa (machacando la semilla, por ejemplo), se exponen las fibras internas de modo que interaccionan formando una red tridimensional (Figura 4). Se ha demostrado que la formación de esta red es más fuerte y presenta mayor efecto en la retención de agua, reducción de obesidad, etc (Samateh et al., 2018).

Las semillas de sésamo, por su parte, están cubiertas por unas fibrillas de celulosa unidas por una matriz compuesta principalmente por hemicelulosa, lignina y pectina (generalmente está formada por fibra insoluble), por lo que hubiera sido recomendable haberlas triturado o molido antes de mezclarlas con el yogur (Montalvo et al., n.d.).

La saciedad es un aspecto muy difícil de cuantificar y generalizar a toda la población,

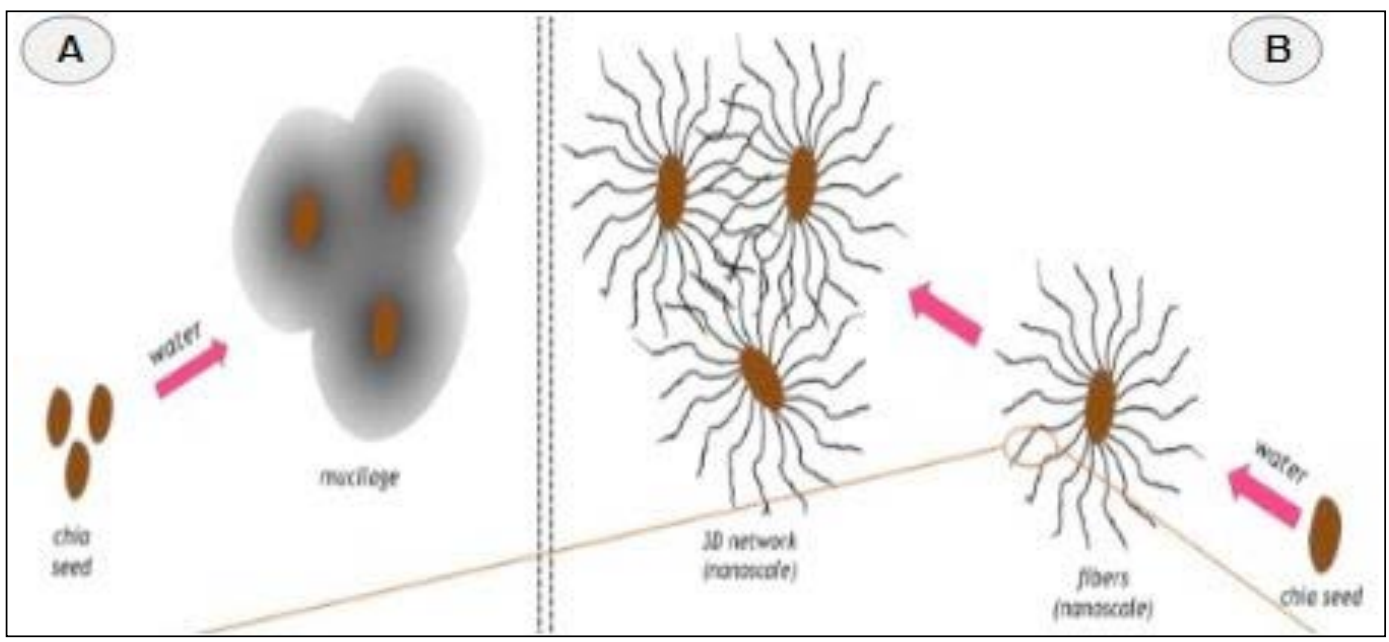

Figura 4: A) gelificación de las semillas de chía (enteras) al unirse con agua; B) gelificación de las semillas de chía sin cubierta, formando una red 3D (extraído de Samateh et al., 2018). 
puesto que no depende de un único factor, sino que son muchos los factores a tener en cuenta, lo que hace muy complejo controlarlos todos.

A pesar de no encontrar diferencias significativas entre los 3 alimentos en estudio, sí se encontraron correlaciones entre las diferentes variables tomadas a los voluntarios y los cuestionarios realizados (tabla 3 ).

La correlación entre la cantidad de grasa (Kg) y la capacidad de desinhibición (comer de forma descontrolada) de una persona a la hora de la comida, es positiva (coeficiente de correlación de 0.6484), indicando que las personas que tiene mayor cantidad de grasa se descontrolan más a la hora de comer. Esta conducta alimentaria no es característica únicamente de la población obesa, sino que la población con normopeso también puede adquirirla, aunque no llegue a engordar. El hecho de que una persona en normopeso coma sin engordar puede deberse a que a la misma vez que aumenta su aporte calórico, aumente el gasto energético (realizando actividad física), sin olvidarnos de la importancia del factor genético
(López-Morales, 2018). Igualmente, existe una correlación entre el IMC y la desinhibición (coeficiente de correlación de 0.5789).

Otra correlación interesante encontrada en este estudio es la existente entre el IMC y la restricción, obteniendo un coeficiente muy cercano a 1. Hay diversos autores que proponen que el aumento del deseo de comer es una situación previa a la restricción, debido a un deterioro del control cognitivo (López-Morales, 2018). Es por esto por lo que las emociones presentan una gran influencia con la conducta alimentaria de cada persona. Esta correlación se puede relacionar con factores psicológicos que suele tener una persona obesa como, por ejemplo, restringirse mucho a la hora de comer, tener adicción por la comida o comer de forma emocional.

Es muy interesante que salieran dos correlaciones contradictorias entre el IMC y la restricción (las personas con mayor IMC son las que más se restringen) y el IMC y la desinhibición (las personas con mayor IMC son las que más se descontrolan con la comida), por lo tanto,

Tabla 3. Correlaciones entre las variables obtenidas.

\begin{tabular}{lll}
\hline Correlaciones & $\mathrm{p}$-valor $(\mathrm{p} \leq 0.05)$ & Coeficiente $(0-1$ o 0 a-1) \\
\hline Kg Grasa y D* & 0.00361 & 0.6484 \\
Kg Grasa y H* & 0.0347 & 0.4998 \\
Kg Músculo y gasto calórico & $2 *\left(10^{\wedge}-16\right)$ & 0.8803 \\
$R^{*}$ y estrés & 0.05 & -0.4441 \\
gasto calórico y estrés & 0.0313 & -0.5081 \\
R y peso (Kg) & 0.01814 & 0.5496 \\
R y IMC & 0.0125 & 0.5753 \\
IMC* y D & 0.0133 & 0.5789 \\
AUC y H & 0.05 & 0.4558 \\
Grasa visceral y años & 0.04156 & 0.4855 \\
D y H & $1.14 *\left(10^{\wedge}-5\right)$ & 0.8426 \\
IMC y D & 0.00195 & 0.6788 \\
IMC y R & 0.0125 & 0.8216 \\
H y AUC* & 0.05 & 0.4558 \\
Años y H & 0.0331 & -0.0481 \\
\hline
\end{tabular}

*D: desinhibición; *H: hambre; *R: restricción; *IMC: índice de masa corporal; *AUC: área bajo la curva 
a mayor IMC mayor restricción y mayor desinhibición. Esto puede ocurrir porque este tipo de personas suelen tener momentos de atracón, donde no se preocupan por la cantidad de comida, seguidos de una conducta compensatoria (restringiéndose mucho más para contrarrestar el atracón) (López-Morales, 2018).

En relación a la cantidad de músculo $(\mathrm{Kg})$ y el gasto calórico (cal), se ha encontrado una correlación muy significativa, encontrando un coeficiente de 0.8803 . Esto era lo esperado, ya que la tasa metabólica de la grasa es muy inferior a la del músculo (2 cal $/ \mathrm{kg}$ versus $6 \mathrm{cal} / \mathrm{kg}$ ) (Wang et al., 2007).

También resulta interesante la correlación negativa que se encontró entre hambre y edad de los participantes (-0.0481), lo que nos indica que de manera significativa que cuanto mayor es una persona, menos sensación de hambre tiene.

\section{CONCLUSIONES}

A pesar de las características nutricionales que proporcionan las semillas de chía y sésamo, y su posible relación con la saciedad, en nuestro estudio no se encontraron diferencias significativas entre los 3 tipos de desayunos. Se debe de tener en cuenta que el factor saciante de las semillas es muy difícil aislar en personas, ya que siempre suelen estar introducidas junto con algunos alimentos como en ensaladas, yogur, etc. Por ello al introducirlas en el desayuno se debe de tener en cuenta el papel saciante del café, de la tostada y del yogur en conjunto.

Por otro lado, sí que encontramos correlaciones interesantes entre las distintas variables tomadas a los sujetos (variables antropométricas y cuestionario TFEQ y PSS).

Son pocos los estudios que relacionan el efecto saciante con algún tipo de semillas. Además, la gran mayoría de ellos son estudios a corto plazo, por ello sería muy interesante analizar su posible efecto a largo plazo a fin de conocer, de una forma más consistente, el efecto saciante de las semillas.

\section{AGRADECIMIENTOS}

Al Ministerio de Economía y Competitividad (España) por el proyecto AGL201678125-R. También al Ministerio de Educación, Cultura y Deporte (España) por la beca predoctoral de D. Planes-Muñoz.

\section{REFERENCIAS BIBLIOGRÁFICAS}

Amin, T., y Mercer, J. G. (2016). Hunger and Satiety Mechanisms and Their Potential Exploitation in the Regulation of Food Intake, Current obesity reports (Vol. 5, Issue 1, pp. 106-112). Springer. https://doi.org/10.1007/ s13679-015-0184-5

Asghar, A., Majeed, M. N., y Akhtar, M. N. (2014). A review on the utilization of sesame as functional food. American Journal of Food and Nutrition, 4(1), 21-34. https://doi. org/10.5251/ajfn.2014.4.1.21.34

Ayaz, A., Akyol, A., Inan-Eroglu, E., Kabasakal Cetin, A., Samur, G., y Akbiyik, F. (2017). Chia seed (Salvia hispanica L.) added yogurt reduces short-term food intake and increases satiety: randomised controlled trial. Nutrition Research and Practice, 11(5), 412-418. https://doi.org/10.4162/nrp.2017.11.5.412

Bach-Faig, A., Berry, E. M., Lairon, D., Reguant, J., Trichopoulou, A., Dernini, S., Medina, F. X., Battino, M., Belahsen, R., Miranda, G., Serra-Majem, L., y Mediterranean Diet Foundation Expert Group. (2011). Mediterranean diet pyramid today. Science and cultural updates. Public Health Nutrition, 14(12A), 2274-2284. https://doi. org/10.1017/S1368980011002515

Blundell, J., De Graaf, C., Hulshof, T., Jebb, S., Livingstone, B., Lluch, A., Mela, D., Salah, S., Schuring, E., Van Der Knaap, H., y Westerterp, M. (2010). Appetite control: Methodological aspects of the evaluation of foods. In Obesity Reviews, (Vol. 11, Issue 3, pp. 251-270). https://doi.org/10.1111/ j.1467-789X.2010.00714.X 
Buckland, G., Bach Faig, A., y Serra Majem, L. (2008). Eficacia de la dieta mediterránea en la prevención de la obesidad. Revista Española de Obesidad, 6(6), 329-339. https://www.researchgate.net/publication/242088165

Carbonneau, É., Royer, M.-M., Richard, C., Couture, P., Desroches, S., Lemieux, S., y Lamarche, B. (2017). Effects of the Mediterranean Diet before and after Weight Loss on Eating Behavioral Traits in Men with Metabolic Syndrome. Nutrients, 9(3), 305. https://doi.org/10.3390/nu9030305

Carranza LE. (2016). Fisiología del apetito y el hambre. Enfermería Investiga. (Vol. 1, Issue 3). https://dialnet.unirioja.es/servlet/ articulo? codigo $=6194254$

Clave, A. (1999). Adaptación preliminar del cuestionario de alimentación de STUNKARD y MESSICK (Three Factor Eating Questionnaire, TFEQ) con una muestra española universitaria, Psicología Conductual, 7(3), 393-416.

Davis, C., Bryan, J., Hodgson, J., y Murphy, K. (2015). Definition of the Mediterranean Diet; a Literature Review. Nutrients, 7(11), 9139-9153. https://doi.org/10.3390/ nu7115459

Dernini, S., y Berry, E. M. (2015). Mediterranean Diet: From a Healthy Diet to a Sustainable Dietary Pattern. Frontiers in Nutrition, 2, 15. https://doi.org/10.3389/ fnut.2015.00015

García-Flores, C. L., Martínez Moreno, A. G., Beltrán Miranda, C. P., Zepeda-Salvador, A. P., y Solano Santos, L. V. (2017). Saciación vs saciedad: Reguladores del consumo alimentario. Revista Medica de Chile, 145(9), 1172-1178. https://doi.org/10.4067/s003498872017000901172

Gibney, M.J., Barr, S.I., Bellisle, F., Drewnowski, A., Fagt, S., Livingstone, B., Masset, G., Varela Moreiras, G., Moreno, L.A., Smith, J., Vieux, F., Thielecke, F., y Hopkins, S., (2018). Breakfast in Human Nutrition:
The International Breakfast Research Initiative. Nutrients, 10: 559. https://doi.org/ doi:10.3390/nu10050559.

Halford, J. C. G., y Harrold, J. A. (2012). Satellite Symposium : Industry and academic partnerships for developing Satiety-enhancing products for appetite control : science and regulation of functional foods for weight management $\dagger$ Proceedings of the Nutrition Society. July 2011. https://doi.org/10.1017/ S0029665112000134

López-Aguilar, X., Manuel Mancilla-Díaz, J., Vázquez-Arévalo, R., Franco-Paredes, K., y Leticia Alvarez-Rayón Ma Trinidad Ocampo Téllez-Girón, G. (2011). Psychometric properties of the Three Factor Eating Questionnaire (TFEQ) Propiedades psicométricas del Cuestionario de Tres Factores de la Alimentación (TFEQ). Revista Mexicana de Trastornos Alimentarios Mexican Journal of Eating Disorders Journal homepage (Vol. 2). http://journals .iztacala.unam.mx/

López-Morales, J. L. (2018). Análisis del comportamiento alimentario y sus factores psicológicos en población universitaria no obesa. Anales de Psicologia, 34(1). https://doi. org/10.6018/analesps.34.1.294341

Montalvo, M., Campos, D., Chirinos, R., y Universidad, A. La. (n.d.). Estudio del pelado enzimatico de la semilla de ajonjoli (Sesanum indicum L). https://smbb.mx/ congresos smbb/veracruz01/TRABAJOS/ AREA_V/CV-41.pdf

Montmayeur, J.-P., y Coutre, J. le. (2009). Fat Detection: Taste, Texture, and Post Ingestive Effects. CRC/Tayor \& Francis. https://books.google.com/ books? $\mathrm{id}=\mathrm{jSb} 7 \mathrm{aQxm9UEC} \&$ pgis $=1$

Organizacion Mundial de la Salud (OMS). (2012). Obesidad y Sobrepeso. Nota Descriptiva $\mathrm{N}^{\circ} 311$. https://www.who.int/es/ news-room/fact-sheets/detail/obesity-andoverweight

Pal, A. (2010). Nutritional, Medicinal and Industrial Uses of Sesame ( Sesamum indi- 
cum L .) Seeds - An Overview. Agriculturae Conspectus Scientificus, 75(4), 159-168

Saeed, F., Qamar, A., Nadeem, M. T., Ahmed, R. S., y Arshad, M. S. (2015). Nutritional composition and fatty acid profile of some promising sesame cultivars. Pakistan Journal of Food Sciences, 25(2), 98-103.

Samateh, M., Pottackal, N., Manafirasi, S., Vidyasagar, A., Maldarelli, C., y John, G. (2018). Unravelling the secret of seed-based gels in water: the nanoscale 3D network formation. Scientific Reports, 8(1), 7315. https://doi. org/10.1038/s41598-018-25691-3

Sociedad Venezolana de Farmacología., L., Sociedad Venezolana de Farmacología Clínica y Terapéutica., M. C., Garrido, M., Carrasco, P., López-Miranda, J., Aparicio, D., Céspedes, V., González, R., Chaparro, R., Angarita, M., WilchesDuran, S., Graterol-Rivas, M., Chacón, J., Cerda, M., Contreras-Velasquez, J., Reina, N., \& Bermúdez, V. (2016). Archivos venezolanos de farmacología y terapéutica. Archivos Venezolanos de Farmacología y Terapéutica (Vol. 35, Issue 4). Sociedad Venezolana de Farmacología y Farmacología Clínica y Terapéutica, Escuela de Medicina José María Vargas, Cátedra De Farmacología. http://www. scielo.org.ve/scielo.php?script=sci_arttext \& pid=S0798-02642016000400004

Torres-Lagunas, M. A., Vega-Morales, E. G., Vinalay-Carrillo, I., Arenas-Montaño, G., Rodríguez-Alonzo, E., Torres-Lagunas, M.
A., Vega-Morales, E. G., Vinalay-Carrillo, I., Arenas-Montaño, G., y Rodríguez-Alonzo, E. (2015). Validación psicométrica de escalas PSS-14, AFA-R, HDRS, CES$\mathrm{D}, \mathrm{EV}$ en puérperas mexicanas con y sin preeclampsia. Enfermería Universitaria, 12(3), 122-133. https://doi.org/10.1016/j. reu.2015.08.001

Tremblay, A., y Bellisle, F. (2015). Nutrients, satiety, and control of energy intake. Applied Physiology, Nutrition, and Metabolism, 40(10), 971-979. https://doi.org/10.1139/ apnm-2014-0549

Verónica, D., y Vliegenthart, Á. (2003). Obesidad: la epidemia del siglo XXI. 14(10).

Vuksan, V., Choleva, L., Jovanovski, E., Jenkins, A. L., Au-Yeung, F., Dias, A. G., Ho, H. V. T., Zurbau, A., y Duvnjak, L. (2017). Comparison of flax (Linum usitatissimum) and Salba-chia (Salvia hispanica L.) seeds on postprandial glycemia and satiety in healthy individuals: a randomized, controlled, crossover study. European Journal of Clinical Nutrition, 71(2), 234-238. https:// doi.org/10.1038/ejen.2016.148

Wang, Z., Heshka, S., Wang, J., Gallagher, D., Deurenberg, P., Chen, Z., \& Heymsfield, S. B. (2007). Metabolically active portion of fat-free mass: a cellular body composition level modeling analysis. American Journal of Physiology. Endocrinology and Metabolism, 292(1), E49-53. https://doi. org/10.1152/ajpendo.00485.2005 